\title{
PHOTOCHEMICAL PROPERTIES OF THE NEW FLAVIN PHOSPHATE OF RHIZOPUS'
}

\author{
SEI TACIIBANA, YUZO NOJIRI, JUIIHI SHIOIDE \\ INI) YOSHIVIK OOTA

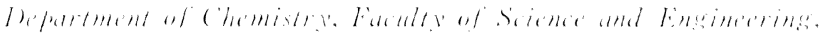

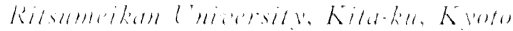

Recoived September 8. 196 iti

It was previously revealed that a new flavin phosphate $\left(\begin{array}{ll}1 & 2)\end{array}\right)$ of Rhizopus, flavin cyclic '2',5'-mononucleotide (3), requiring an unknown phosphate donor other than ATP, Cori ester and p-nitrophenylphosphate (4 7$)$, had no growth-promoting activity for Lactotuaillus casei ATCC No. 7469 (8).

This finding prompted us to investigate the photochemical properties of FMN'. Consequently, it was found that the fluorescence-pH curve of FMN' was markedly different from those of FMN and riboflavin, and that F.MN' not only failed to react as a sensitizer in the dye-sensitized photopolymerization of acrylamide under some conditions, hut appatrently inhibited the photopolymerization by fiMN or riboflavin,

\section{FXPERIMENTMI}

\section{Flarins and ()har Materials}

FMN was enzymatically synthesized from riboflavin by the dry mycelia of Rhisopus or.sear and Rh. jadmicus as described hy the authors. Riboflavin and F.MN were purchased from Nakarai Chemicals lot. Arryamide and other inorganic salts were obtained from the Chemicals.

\section{Fluorescence-pll ('ura's}

Fluorescence-pll curves were routinely obtained by means of Schimazu-Kotaki Microphoto-reflecting fluorometer and Ilitachi Model M 3 pll meter and the percentage fluorescence was measured at the pll range of 1.2 to 11.0 in $1.33 \times 10^{-6} .1 /$ flavin solution at room temperature. 25

\section{Photosensitised P'olymerisation}

The procedure of photopolymerization was a modification of the technique first described by ()ster al al (9) 11), using ()stwald viscometer (43.4 sec meter). An aliquot of $4 \mathrm{ml}$ flavin solution was added to $4 \mathrm{ml}$ of 50 on (by weight) acrylamide solution in a test tube. The mixture was shaken for 1 minute moderately. and put into the ()stwald viscometer, immersed in an incubater at 30. After"?

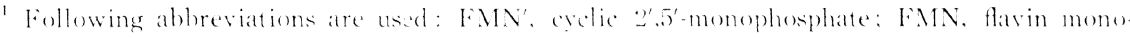
nucleotide.

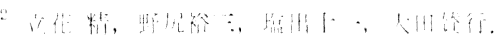


minutes, the flow velocity of the mixture was measured prior to irradiation.

After taking out of the incubator, the globe of the viscometer was irradiated through the $5 \mathrm{~cm}$-diameter window of GS-H400 type high pressure mercury lamp at $29 \mathrm{~cm}$ distance from the center of the lamp. After 3 minute-irradiation without shaking, immediately the viscometer was immersed in the incubator at $30^{\circ}$ for 2 minutes, and the flow velocity of the irradiated mixture was measured in the incubator at $30^{\circ}$. This procedure was repeated with about 5 minute-interval. The irradiation time in Figs. 2 and 3 is the summation of succesive irradiation time. The temperature of alcoholic thermometer at the irradiated position was about 29 \% It was 14,000 lux at the same position.

\section{RESULTS}

\section{Fluorescence-pH Curve}

The fluorescence-pH curve of $\mathrm{FMN}^{\prime}$ was very different from those of riboflavin and FMN as shown in Fig. 1. In the curve two peakes were shown at $\mathrm{pH} 4.3$ and 7.3. The percentage fluorescence at $\mathrm{pH} 4.3$ and 7.3 were 95 and $85 \%$ of riboflavin and FMN, respectively. By the way, the fluorescence-pH curve of FAD has, according to the literature, one peak of about $60 \%$ of riboflavin or FMN at pH 3 .

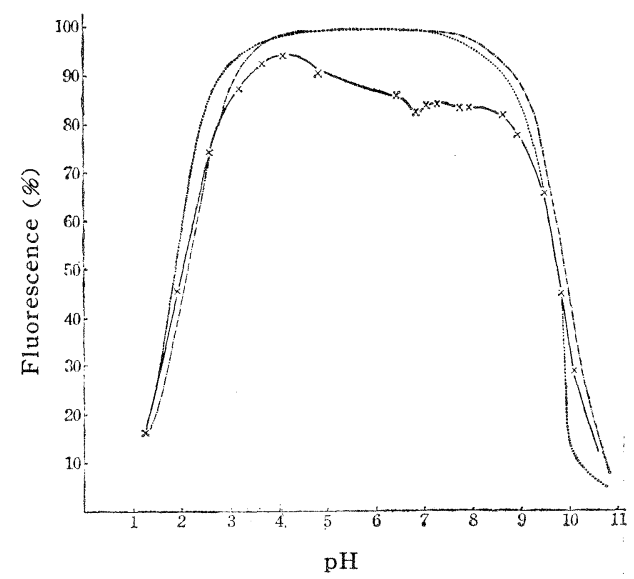

FIG. 1 Fluorescence-pH Curve of $F M N^{\prime}$

-, new flavin; -.., riboflavin; -.-, FMN.

\section{Photosensitized Polymerization}

The results of the photosensitized polymerization reaction of acrylamide solution with $2.66 \times 10^{-4} \mathrm{MFMN}$, riboflavin and $\mathrm{FMN}^{\prime}$ as a photosensitizer, respectively, were shown in Fig. 2. The flow velocity of the irradiated reaction mixture containing FMN was 67, 70,91 and 146 seconds after irradiation for 0,6, 9 and 12 minutes, respectively. After addition of riboflavin, the flow velocity was 67,68 , 78, 98 and 143 seconds after irradiation for $0,6,9,12$ and 15 minutes. On the contrary, when $\mathrm{FMN}^{\prime}$ was used as the photosensitizer, no photopolymerization was observed in 36 minutes. This result suggested that $\mathrm{FMN}^{\prime}$ could not react as a 


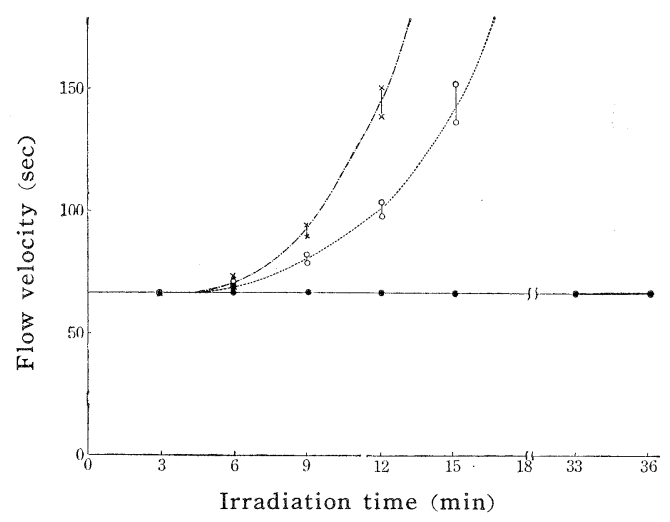

FIG. 2 Photo-sensitized Polymerization of Acrylamide by Riboflavin $F M N$ and New Flavin $\left(F M N^{\prime}\right)$

--, new flavin; -.., riboflavin; -.-, FMN.

sensitizer in the dye-sensitized photopolymerization under the conditions employed.

To obtain further informations about the behavior of $\mathrm{FMN}^{\prime}$, the effect of co-addition of $1.33 \times 10^{-4} M \mathrm{FMN}^{\prime}$ and $\mathrm{FMN}$ as the sensitizer on the photopolymerization of acrylamide was investigated. The co-addition resulted in no increase of viscosity of acrylamide solution after 24 minute-irradiation. The result was shown in Fig. 3 accompanied with the results of photopolymerization with 0.665 , $1.33,2$, and $2.66 \times 10^{-4} M$ FMN. From these results, FMN' seemed to inhibit the photopolymerization of acrylamide with FMN.

Furthermore, the effect of $\mathrm{FMN}^{\prime}$ on the photopolymerization with riboflavin was investigated. As shown in Fig. 4, if compared with the five flow velocity curves obtained by the photopolymerization with $0.53,0.665,1.33,2$, and $2.66 \times$ $10^{-4} \mathrm{M}$ riboflavin, it was apparently observed that the photopolymerization with

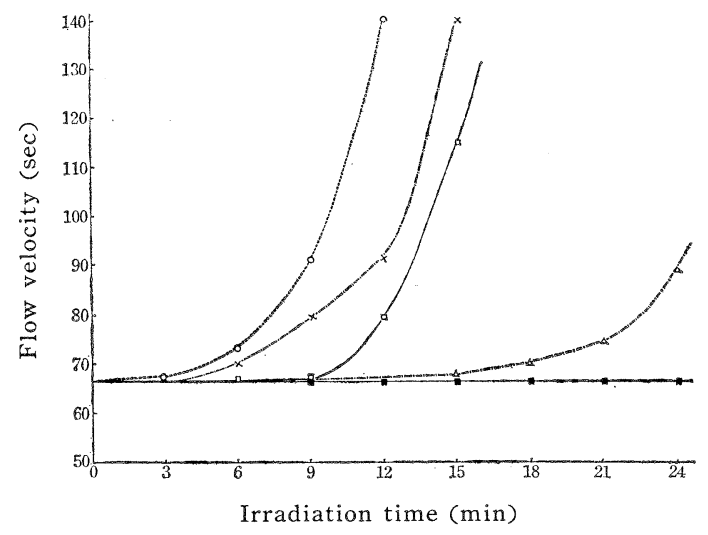

FIG. 3 Inhibitory Effect of FMN' on Photosensitized Polymerization by FMN

○, FMN, $2.66 \times 10^{-4} M ; \times, \mathrm{FMN}, 2 \times 10^{-4} \mathrm{M} ; \quad \square, \mathrm{FMN}, 1.33 \times 10^{-4} \mathrm{M}$;

$\triangle$ FMN, $0.665 \times 10^{-4} M ; \boldsymbol{\square}, \mathrm{FMN}, 1.33 \times 10^{-4} \mathrm{M}+$ new flavin, $1.33 \times 10^{-4} M$. 
riboflavin was inhibited by $\mathrm{FMN}^{\prime}$, too. It therefore seemed quite reasonable to postulate that the inhibitory effect of $\mathrm{FMN}^{\prime}$ was derived from the conformation of isoalloxazine ring affected by the $2^{\prime}, 5^{\prime}$-cyclic monophosphate of $\mathrm{FMN}^{\prime}$.

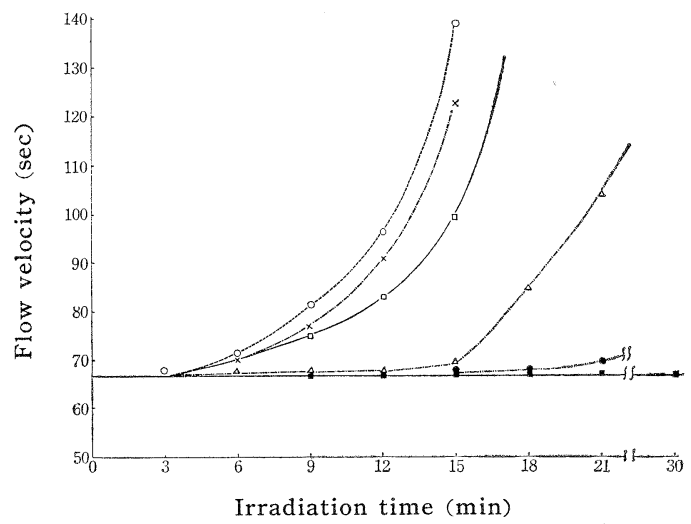

FIG. 4 Inhibitory Effect of $F M N^{\prime}$ on Photosensitized Polymerization by Riboflavin

O, riboflavin, $2.66 \times 10^{-4} \mathrm{M} ; \times$, riboflavin, $2 \times 10^{-4} \mathrm{M} ; \square$, riboflavin, $1.33 \times 10^{-4} \mathrm{M} ; \triangle$, ribollavin, $0.665 \times 10^{-4} \mathrm{M} ; \bullet$, riboflavin, $0.53 \times 10^{-4} \mathrm{M}$;

E, riboflavin, $1.33 \times 10^{-4} M+$ new flavin, $1.33 \times 10^{-4} M$.

\section{Photolysis}

Although it was expected that the photolysis of $\mathrm{FMN}^{\prime}$ during irradiation might be one of the causes of the inhibition of photosensitized polymerization, it was experimentally revealed that $\mathrm{FMN}^{\prime}$ was more stable to the irradiation by day light and mercury lamp than FMN. The details of the photolysis will appear in another paper. The investigation of the mechanism of inhibition is in progress.

\section{DISCUSSION}

It must be emphasized that the $\mathrm{FMN}^{\prime}$ having no growth-promoting activity for Lactobacillus casei was not only inactive as a photosensitizer, but also inhibitory for the photopolymerization of acrylamide with FMN and riboflavin. The difference between the fluorescence-pH curves of $\mathrm{FMN}^{\prime}$ and $\mathrm{FMN}$ may reflect the change of conformation due to the interaction between the $2^{\prime}, 5^{\prime}$-cyclic mononucleotide and isoalloxazine in $\mathrm{FMN}^{\prime}$ molecule. The interaction, even if not strong enough to produce the difference of ultraviolet absorption spectrum of $\mathrm{FMN}^{\prime}$, might cause the photopolymerization to be inhibited, and cause the photolysis to be delayed. Moreover, the interaction might be concerned with the stability towards the phosphate liberating enzymes.

Further investigations of the interrelations between the enzymatic and photochemical properties of riboflavinylglucoside mononucleotide and riboflavin cyclic diphosphate, as well as $\mathrm{FMN}^{\prime}$ are in progress. 


\section{SUMMARY}

1. The new flavin phosphate of Rhizopus, flavin cyclic $2^{\prime}, 5^{\prime}$-mononucleotide $\left(\mathrm{FMN}^{\prime}\right)$, was revealed to have the medium shape of fluorescence-pH curve as compared to those of FMN and FAD, of which percentage fluorescence was 95 and $83 \%$ of $\mathrm{FMN}$ at $\mathrm{pH} 4.3$ and $\mathrm{pH} 6.9$ respectively.

2. $\mathrm{FMN}^{\prime}$ failed to react as a sensitizer in dye-sensitized photopolymerization of acrylamide.

3. Equal amount of $\mathrm{FMN}^{\prime}$ co-added with FMN or riboflavin inhibited the dyesensitized photopolymerization of acrylamide.

4. FMN' underwent photolysis more slowly than FMN in some extent.

5. These photochemical properties may be caused by conformation derived from such primary structure.

\section{REFERENCE}

1. Tachibana, S., Vitamins 22, 291 (1961); J. Vitaminol. 7, 294 (1961).

2. Tachibana, S., Siode, J., and Matsuno, S., Vitamins 27, 210 (1963); J. Vitaminol. 9, 197 (1963).

3. Tachibana, S., Abstract of the 36th Annual Meeting of the Jap. Biochem. Soc., Seikagaku 35, 616 (1963).

4. Tachibana, S., Vitamins 29, 209 (1964).

5. Tachibana, S., Siode, J., Tamura, M., Oosaki, T., Yamaguchi, H., Kusida, S., Yamauchi, K., Kuriyama, T., Takenaka, H., Goda, Y., Matsui, E., and Matuno, S., Symposium on Enzymchem. 16, 271 (1964).

6. Tachibana, S., Tamura, M., and Siode, J., Abstract of the 16th Annual Meeting of the Vitamin Soc. of Jap., Vitamins 29, 553 (1964)

7. Tachibana, S., and Tamura, M., Abstract of the 37th Annual Meeting of the Jap. Biochem. Soc., Seikagaku 36, 642 (1964).

8. Tachibana, S., Oosaki, T., and Siode, J., Vitamins 29, 534 (1964).

9. Oster, G., Nature 173, 300 (1954).

10. Oster, G., and Mizutani, Y., J. Polymer Sci. 22, 173 (1956).

11. Oster, G., and Adelmann, A. H., J. Amer. Chem. Soc. 78, 913 (1956). 